\title{
Quantitative and Qualitative Comparison of 4D-DSA with 3D-DSA Using Computational Fluid Dynamics Simulations in Cerebral Aneurysms
}

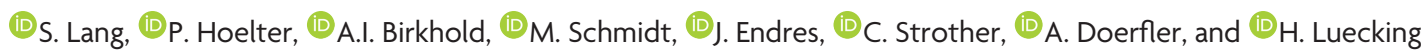

\begin{abstract}
BACKGROUND AND PURPOSE: 4D-DSA allows time-resolved 3D imaging of the cerebral vasculature. The aim of our study was to evaluate this method in comparison with the current criterion standard 3D-DSA by qualitative and quantitative means using computational fluid dynamics.
\end{abstract}

\begin{abstract}
MATERIALS AND METHODS: 3D- and 4D-DSA datasets were acquired in patients with cerebral aneurysms. Computational fluid dynamics analysis was performed for all datasets. Using computational fluid dynamics, we compared 4D-DSA with 3D-DSA in terms of both aneurysmal geometry (quantitative: maximum diameter, ostium size [OZ1/2], volume) and hemodynamic parameters (qualitative: flow stability, flow complexity, inflow concentration; quantitative: average/maximum wall shear stress, impingement zone, low-stress zone, intra-aneurysmal pressure, and flow velocity). Qualitative parameters were descriptively analyzed. Correlation coefficients ( $r, P$ value) were calculated for quantitative parameters.
\end{abstract}

RESULTS: 3D- and 4D-DSA datasets of 10 cerebral aneurysms in 10 patients were postprocessed. Evaluation of aneurysmal geometry with 4D-DSA $\left(r_{\text {maximum diameter }}=0.98, P_{\text {maximum diameter }}<.001 ; r_{\text {OZ1/OZ2 }}=0.98 / 0.86, P_{\text {OZ1/OZ2 }}<.001 / .002 ; r_{\text {volume }}=0.98, P_{\text {volume }}<.001\right)$ correlated highly with 3D-DSA. Evaluation of qualitative hemodynamic parameters (flow stability, flow complexity, inflow concentration) did show complete accordance, and evaluation of quantitative hemodynamic parameters $\left(r_{\text {average/maximum wall shear stress diastole }}=0.92 / 0.88\right.$, $P_{\text {average/maximum wall shear stress diastole }}<.001 / .001 ; r_{\text {average/maximum wall shear stress systole }}=0.94 / 0.93, P_{\text {average/maximum wall shear stress systole }}<$ $.001 / .001 ; r_{\text {impingement zone }}=0.96, P_{\text {impingement zone }}<.001 ; r_{\text {low-stress zone }}=1.00, P_{\text {low-stress zone }}=.01 ; r_{\text {pressure diastole }}=0.84, P_{\text {pressure diastole }}=$ $\left..002 ; r_{\text {pressure systole }}=0.9, P_{\text {pressure systole }}<.001 ; r_{\text {flow velocity diastole }}=0.95, P_{\text {flow velocity diastole }}<.001 ; r_{\text {flow velocity systole }}=0.93, P_{\text {flow velocity systole }}<.001\right)$ did show nearly complete accordance between 4D- and 3D-DSA.

CONCLUSIONS: Despite a different injection protocol, 4D-DSA is a reliable basis for computational fluid dynamics analysis of the intracranial vasculature and provides equivalent visualization of aneurysm geometry compared with 3D-DSA.

ABBREVIATIONS: AWSS = average wall shear stress; $C F D=$ computational fluid dynamics; $d_{\max }=$ maximum diameter; $\mathrm{IZ}=$ impingement zone; $\mathrm{LSZ}=$ low-stress zone; MWSS = maximum wall shear stress; $\mathrm{OZ}=$ ostium size; $r=$ correlation coefficient; $\mathrm{V}=$ flow velocity; WSS $=$ wall shear stress

D we to its accuracy, cerebral angiography consisting of $2 \mathrm{D}$ and 3D-DSA is regarded as the criterion standard for visualization of the cerebral vasculature. ${ }^{1,2}$ Despite the high image qual-

Received April 9, 2019; accepted after revision July 1.

From the Department of Neuroradiology (S.L., P.H., M.S., J.E., A.D., H.L.), University of Erlangen-Nuremberg, Erlangen, Germany; Siemens Healthcare GmbH (A.I.B.), Erlangen, Germany; and Department of Radiology (C.S.), Clinical Sciences Center, University of Wisconsin School of Medicine and Public Health, Madison, Wisconsin.

The Department of Neuroradiology, University of Erlangen-Nuremberg has a research agreement with Siemens Healthcare GmbH, Erlangen, Germany. Stefan Lang and Philip Hoelter contributed equally to this publication.

Please address correspondence to Stefan Lang, MD, Department of Neuroradiology, University of Erlangen-Nuremberg, Schwabachanlage 6, 91054 Erlangen,

Germany; e-mail: Stefan.Lang3@uk-erlangen.de

http://dx.doi.org/10.3174/ajnr.A6172 ity of 2D-DSA and the opportunity for 3D visualization of the vasculature using 3D-DSA, both methods have limitations. On the one hand, 2D-DSA offers a dynamic, high-resolution illustration of vessels in selected projections. On the other hand, delineation of anatomic details might be impaired by exclusive use of 2D-DSA. ${ }^{3}$ Whereas 2D-DSA has overlap of arterial and venous structures, 3D-DSA is limited by its lack of temporal resolution. Hence, especially in complex pathologies, 2D-DSA should be combined with 3D-DSA to improve the validity of cerebral angiography.

Recently a novel method has been implemented ${ }^{4}$ that offers both static and time-resolved 3D volumes (4D-DSA). 4D-DSA has become a reliable method for improved visualization of vascular anatomy that shows excellent agreement with 2D-DSA. ${ }^{5-8}$ Despite 3D- and 4D-DSA both being based on the rotational ac- 
quisitions of mask and fill projection images, there are relevant technical differences between 3D- and 4D-DSA. For realization of time-resolved 3D imaging, a different acquisition protocol is required. Compared with 3D-DSA, 4D-DSA consists of a longer scanning time $(4 \mathrm{D}-\mathrm{DSA}=12$ seconds versus $3 \mathrm{D}-\mathrm{DSA}=5 \mathrm{sec}-$ onds) and a higher rotational angle (rotational angle 4D-DSA = $260^{\circ}$ versus rotational angle $3 \mathrm{D}-\mathrm{DSA}=200^{\circ}$ ) to record the inand outflow of contrast medium. If one took into account that $4 \mathrm{D}$-DSA represents a new and different approach for 3D visualization of vasculature, the question of noninferiority of the static reconstructions of 4D-DSA compared with 3D-DSA comes automatically to the fore. Because of high intra- and interrater inconsistencies due to different windowing, even "simple" measurements of, for example, the height and length of an aneurysm in 2 different datasets, are challenging. Thus, an objective method for comparing both techniques would be desirable.

In this context, computational fluid dynamics (CFD) analysis of cerebral vasculature might be helpful. Despite the controversial discussion on its clinical relevance, ${ }^{9}$ CFD is considered an established method to evaluate intra-aneurysmal hemodynamics. ${ }^{10,11}$ Whereas 3D-DSA is commonly used as a basis for CFD analysis of cerebral aneurysms, ${ }^{12,13}$ there are no data regarding the qualification of 4D-DSA datasets for CFD. Considering that the geometry of the vascular structure has a significant influence on the deduction of flow parameters, ${ }^{14-17}$ we concluded that CFD analysis of 1 aneurysm based on both 3D- and 4D-DSA could be useful for the evaluation of the static reconstruction of a $4 \mathrm{D}$-DSA dataset. If one assumes that established flow parameters (eg, wall shear stress and so forth) represent the vascular geometry in aneurysms, CFD might be a suitable method to compare the quality of 3D imaging of 4D-DSA with that of 3D-DSA.

In this article, we present our initial experience with 4D-DSA for the calculation of typical CFD parameters. Our aim was to evaluate $4 \mathrm{D}$-DSA in comparison with 3D-DSA using CFD regarding quantitative and qualitative parameters.

\section{MATERIALS AND METHODS Patients}

Patients with cerebral aneurysms who underwent both a $3 \mathrm{D}$ DSA at the diagnostic stage and a 4D-DSA immediately before the intervention due to optimization of working projections (eg, in case of different head positioning) were included in this study retrospectively.

\section{Data Acquisition and Postprocessing}

Angiography (including 3D-DSA and 4D-DSA) was performed using a biplane flat panel detector angiographic system (Artis zee biplane; Siemens, Erlangen, Germany). By standard angiographic methods (via the transfemoral route), a diagnostic catheter $(5 \mathrm{~F})$ was positioned in the proximal internal carotid artery or in the vertebral artery to obtain standard projections (2D-DSA). Additionally, 3D- and 4D-DSA datasets were acquired using commercially available angiographic applications (12-second syngo Dyna 4D and 5-second DSA; Siemens) as previously described. ${ }^{8}$ The datasets were postprocessed with commercially available software (Dyna 4D; Siemens). According to standardization, we used established reconstruction parameters for both 3D- and 4D-
Inlet Velocity Waveform

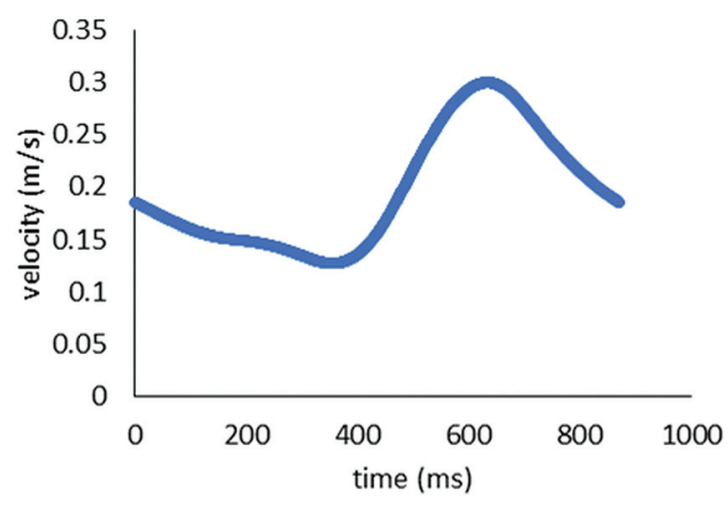

FIG 1. Flow waveform at the inflow representing the inflow conditions used for CFD analysis. A homogeneous velocity profile across the complete inflow cross-section with the velocities given by the inflow curve was applied as inflow boundary conditions. The outflow boundary conditions were defined as zero pressure.

DSA datasets (kernel type: "edge enhanced"; image characteristics: "smooth"; mode of reconstruction: "subtracted"; $512 \times 512$ image matrix; isotropic voxel size $=0.15 \mathrm{~mm}$ ). To provide comparability with 3D-DSA, we used the static $3 \mathrm{D}$ reconstruction of 4D-DSA exclusively for CFD analysis.

\section{CFD}

The simulation was performed on a research prototype (Siemens). ${ }^{18}$ Both $3 \mathrm{D}$ - and $4 \mathrm{D}$-DSA volumes were cropped to the ROI (ie, the aneurysm and parent vessels). Intensity thresholding was used for segmenting the vessels in the volumetric domain. Threshold values were chosen empirically to obtain comparable vessel diameters and aneurysm volumes. In case of intraluminal contrast inhomogeneities within the processed volume (eg, missing contrast caused by crossflow via a nearby vessel), the segmentation was manually corrected to allow proper flow simulations. First, voxel-based representation was transferred to a mesh representation. Second, inlets and outlets, which describe areas of blood entering and leaving the simulated domain, were specified for vessels proximal and distal to the aneurysm. A homogeneous velocity profile across the complete inflow cross-section with the velocities given by the inflow curve (Fig 1) was applied as a boundary condition for the inlets. Blood entered the simulated domain with an average flow velocity of $0.196 \mathrm{~m} / \mathrm{s}$, varying between 0.127 and $0.300 \mathrm{~m} / \mathrm{s}$. Zero pressure was defined as outflow boundary conditions. For the hemodynamic simulation itself, the simulated blood was modeled as Newtonian fluid with a viscosity of 0.004 $\mathrm{Pa} \cdot \mathrm{s}$ and a density of $1000 \mathrm{~kg} / \mathrm{m}^{3}$. The heart rate was 69 beats per minute or 0.87 seconds for 1 heart cycle, respectively.

\section{Data Evaluation}

All 3D- and 4D-DSA datasets were anonymized and stored in random order. Evaluation of the acquired 3D-DSA and 4D-DSA datasets was performed by 2 experienced neuroradiologists in a consensus reading, blinded to clinical information.

\section{Image Quality}

All 3D- and 4D-DSA datasets were evaluated for parameters comprising image and diagnostic quality (eg, movement artifacts). 


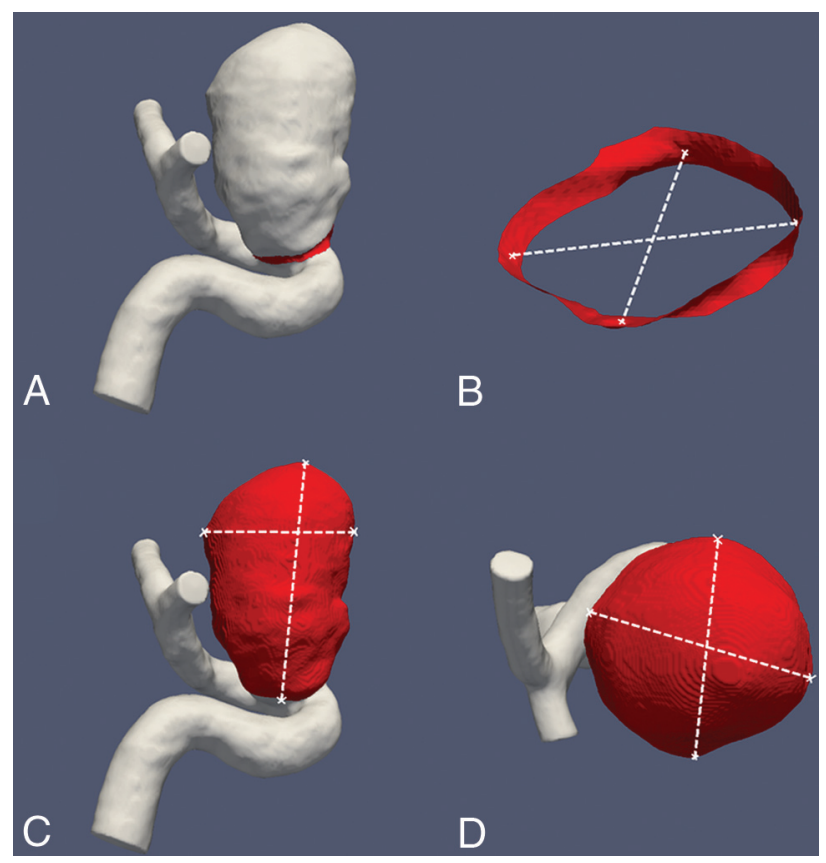

FIG 2. Images exemplarily show measurements of the aneurysmal ostium $(A$ and $B$ ) and the aneurysm itself $(C$ and $D)$ for calculation of the ostium size, maximum diameter, and aneurysmal volume.

The quality of the acquired 3D- and 4D-DSA datasets was assessed in a consensus reading using a 5-fold scaled grading system: $4=$ excellent (high contrast, no artifacts); $3=\operatorname{good}$ (high contrast, minimal artifacts, eg, due to movement or metallic implants); $2=$ compromised (eg, noticeable movement artifacts and/or reduced homogeneity of the vessel contrast); 1 = heavily compromised (low contrast and/or strong movement artifacts); $0=$ not diagnostic (vasculature is not differentiable due to heavy artifacts and/or missing contrast).

\section{Fluid Dynamics Analysis}

Geometry, Pressure, and Flow Velocity. Quantitative morphologic aneurysmal parameters comprising maximum diameter $\left(\mathrm{d}_{\max }\right)$ (in millimeters), maximum volume (in cubic millimeters), and ostium size $\left(\mathrm{OZ}_{1} / \mathrm{OZ}_{2}\right.$ in millimeters) were analyzed in all $3 \mathrm{D}$ - and $4 \mathrm{D}$-DSA datasets (Fig 2). Intra-aneurysmal pressure (P) (in pascals; mean of all elements) and intra-aneurysmal flow velocity (V) (in millimeters/second, mean) during systole and diastole have been additionally analyzed for all 3D- and 4D-DSA datasets.

Hemodynamics. According to Cebral et al, ${ }^{10}$ we assessed both $3 \mathrm{D}$ - and 4D-DSA datasets regarding the following hemodynamic parameters:

- Flow complexity: Intra-aneurysmal flow has been analyzed regarding the configuration of the recirculation zone. Simple flow means a single recirculation zone within the aneurysm. Complex flow patterns are indicated by flow divisions within the aneurysm and feature more recirculation zones.

- Flow stability: Intra-aneurysmal flow is regarded as stable if there is no change of the flow pattern during 1 cardiac cycle.

- Inflow concentration: Concentrated inflow streams pene- trate deeply into the aneurysmal sac and are thin in the main flow direction. Diffuse inflow is defined as thick flow streams (compared with the aneurysmal neck) that disperse quickly once they enter the aneurysmal sac.

- Wall shear stress: Because WSS is seen as one of the most important parameters regarding the initiation of cerebral aneurysms, ${ }^{19}$ average and maximum wall shear stress (AWSS, MWSS) have been analyzed for systole and diastole (in pascals), respectively. Taking different WSS values into account, we specified 2 different zones of the aneurysmal wall.

- Impingement zone: The impingement zone (IZ) is considered a region of the aneurysmal sac where the inflow streams impact the aneurysmal wall and change their direction. The area of the impingement zone (in cubic millimeters) has been defined as the area with WSS of $>80 \%$ (in pascals) of the maximum WSS and was assessed for both 3D- and 4D-DSA.

- Low-stress zone: The low-stress zone (LSZ) is considered an area of the aneurysmal wall where no impact of inflow streams on the aneurysmal wall is detectable. The low-stress zone was defined as the area (in cubic millimeters) with WSS values of $<0.4 \mathrm{~Pa}$ at the end of the diastole, and it was assessed for both 3D- and 4D-DSA.

\section{Statistical Analysis}

All statistical analyses were performed using commercially available software (SPSS Statistics, Version 19; IBM, Armonk, New York). Qualitative parameters (eg, flow complexity, flow stability, inflow concentration, and so forth) were analyzed using descriptive statistics only. Quantitative parameters (ostium size, aneurysmal diameter and volume, intra-aneurysmal pressure, flow velocity, WSS, impingement zone, low-stress zone, and so forth) were tested for normal distribution using the Kolmogorov-Smirnov test. Correlation of quantitative parameters was analyzed by the Spearman rank correlation coefficient $(r)$. A $P$ value $<.05$ was considered statistically significant.

\section{RESULTS}

\section{Patients}

In total, 10 pairs of 3D-DSA and 4D-DSA datasets were acquired in 10 patients with 1 incidental aneurysm each ( 6 women and 4 men; mean age, $52.3 \pm 8.2$ years; age range, $41-78$ years). Aneurysm location was in both the anterior and the posterior circulations $(\mathrm{ICA}=6, \mathrm{MCA}=2$, basilar artery $=2)$.

\section{Image Quality}

Both reviewers completely agreed regarding image quality. All $3 \mathrm{D}$ - and all 4D-DSA datasets were rated as diagnostic (3D-DSA: grade $4=10$; 4 D-DSA: grade $4=10$ ) .

Geometry, Pressure, and Flow Velocity. There was no significant difference regarding geometry between $3 \mathrm{D}$ - and $4 \mathrm{D}$-DSA in terms of maximum diameter $\left(\mathrm{d}_{\max }\right.$ 3D-DSA $=13.11 \pm 5.7 \mathrm{~mm}$; $\left.\mathrm{d}_{\max 4 \mathrm{D}-\mathrm{DSA}}=13.08 \pm 5.51 \mathrm{~mm} ; r_{\mathrm{dmax}}=0.988 ; P<.001\right)$, maximum volume (volume (vD-DSA $=721 \pm 848 \mathrm{~mm}^{3}$; volume $_{4 \mathrm{D}-\mathrm{DSA}}=$ $\left.698 \pm 813 \mathrm{~mm}^{3} ; r_{\text {volume }}=0.976 ; P<.001\right)$, and ostium size $\left(\mathrm{OZ}_{1 / 3 \mathrm{D}-\mathrm{DSA}}=5.67 \pm 2.31 \mathrm{~mm}, \mathrm{OZ}_{2 / 3 \mathrm{D}-\mathrm{DSA}}=5.23 \pm 2.07 \mathrm{~mm}\right.$; $\mathrm{OZ}_{1 / 4 \mathrm{D}-\mathrm{DSA}}=5.71 \pm 5.42 \mathrm{~mm}, \mathrm{OZ}_{2 / 4 \mathrm{D}-\mathrm{DSA}}=5.34 \pm 2.09 \mathrm{~mm}$; 
Table 1: Aneurysmal geometry

\begin{tabular}{lccc}
\hline \multicolumn{1}{c}{ Parameter } & 3D-DSA (Median, IQR) & 4D-DSA (Median, IQR) & $\boldsymbol{r}$ \\
\hline Max. diameter $(\mathrm{mm})$ & $11(9-20)$ & $11(9-20)$ & $0.988(P<.001)$ \\
Max. volume $\left(\mathrm{mm}^{3}\right)$ & $356(161-1196)$ & $381(159-1065)$ & $0.976(P<.001)$ \\
Ostium size $_{1}(\mathrm{~mm})$ & $5(4-7)$ & $5(4-7)$ & $0.976(P<.001)$ \\
Ostium size $_{2}(\mathrm{~mm})$ & $5(4-6)$ & $5(4-6)$ & $0.857(P=.002)$ \\
\hline
\end{tabular}

Note:-Max. indicates maximum; IQR, interquartile range.

Table 2: Aneurysmal pressure, flow velocity, and hemodynamics

\begin{tabular}{lccc}
\multicolumn{1}{c}{ Parameter } & 3D-DSA (Mean) & 4D-DSA (Mean) & \multicolumn{1}{c}{$\boldsymbol{r}$} \\
\hline Intra-aneurysmal pressure $_{\text {systole }}(\mathrm{Pa})$ & $502.8 \pm 242.24$ & $492.2 \pm 300.07$ & $0.903(P<.001)$ \\
Intra-aneurysmal pressure $_{\text {diastole }}(\mathrm{Pa})$ & $189.3 \pm 99.13$ & $181.4 \pm 114.4$ & $0.842(P=.002)$ \\
Flow velocity $_{\text {systole }}(\mathrm{mm} / \mathrm{s})$ & $80.8 \pm 50.94$ & $74.89 \pm 48.02$ & $0.930(P<.001)$ \\
Flow velocity $_{\text {diastole }}(\mathrm{mm} / \mathrm{s})$ & $31.61 \pm 22.2$ & $29.9 \pm 21.45$ & $0.952(P<.001)$ \\
AWSS $_{\text {systole }}(\mathrm{Pa})$ & $0.933 \pm 1.02$ & $0.835 \pm 0.853$ & $0.936(P<.001)$ \\
AWSS $_{\text {diastole }}(\mathrm{Pa})$ & $0.26 \pm 0.28$ & $0.24 \pm 0.25$ & $0.918(P<.001)$ \\
MWSS $_{\text {systole }}(\mathrm{Pa})$ & $5.95 \pm 1.83$ & $5.63 \pm 1.64$ & $0.927(P<.001)$ \\
MWSS $_{\text {diastole }}(\mathrm{Pa})$ & $1.84 \pm 0.73$ & $1.78 \pm 0.69$ & $0.879(P=.001)$ \\
${\text { IZ }\left(\mathrm{mm}^{2}\right)}^{\text {LSZ }_{\left(\mathrm{mm}^{2}\right)}}$ & $12.4 \pm 14.2$ & $8.9 \pm 6.81$ & $0.957(P<.001)$ \\
\hline
\end{tabular}

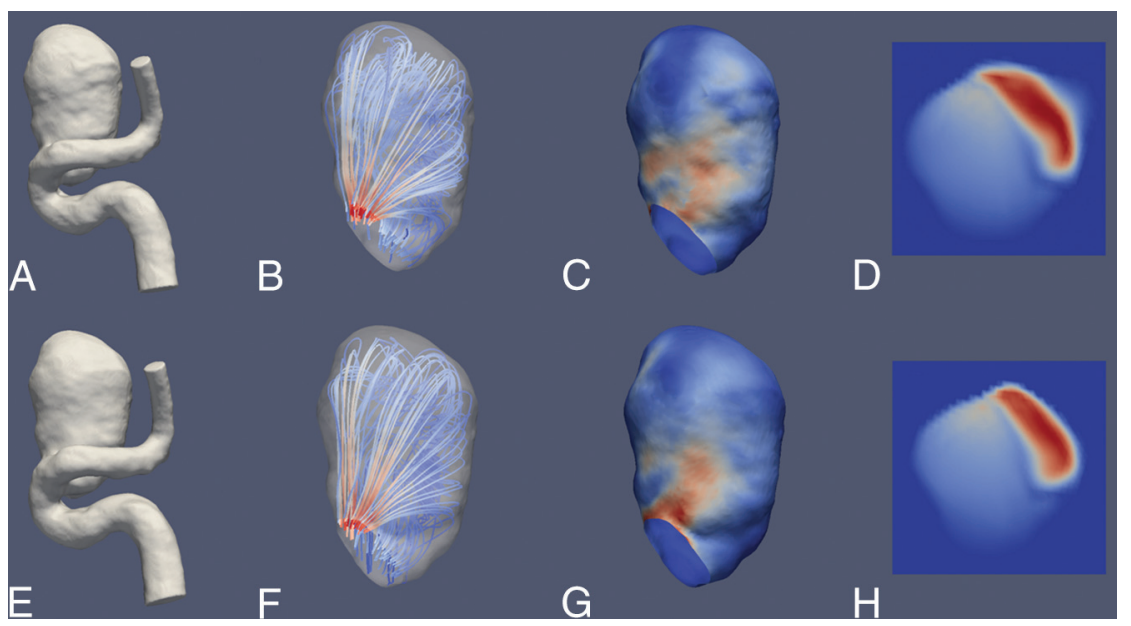

FIG 3. Illustrative case 1 . CFD simulations show a saccular aneurysm of the ICA. Upper row $(A-D)$ and lower row $(E-H)$ show reconstruction results derived from a 3D-DSA and a 4D-DSA dataset, respectively. $A$ and $E, 3 D$ view of the aneurysm using a volume-rendering technique and demonstrating comparable aneurysmal morphology. $B$ and $F$, A color-coded visualization of intra-aneurysmal flow using streamlines (red and blue indicate high- and low-velocity magnitudes). The peak velocities show a laminar distribution along the longitudinal axis. $C$ and $G$, In concordance, note maximum systolic wall shear stress (red and blue indicate high and low WSS) in the corresponding area. $D$ and $H$, The aneurysmal ostium, the associated flow conditions, and the corresponding areas with high-velocity magnitude (red and blue indicate high- and low-velocity magnitude). Overall, the congruence of hemodynamic characteristics between 3D- and 4D-DSA shows geometric accordance between both techniques.

$\left.r_{\mathrm{OZ} 1}=0.976, P<.001 ; r_{\mathrm{OZ} 2}=0.857, P=.002\right)$. There was no significant difference between $3 \mathrm{D}$ - and $4 \mathrm{D}$-DSA regarding intraaneurysmal pressure (systole: $\mathrm{P}_{\text {mean 3D-DSA }}=502.8 \pm 242.24 \mathrm{~Pa}$; $\mathrm{P}_{\text {mean } 4 \mathrm{D}-\mathrm{DSA}}=492.2 \pm 300.07 \mathrm{~Pa}$; diastole: $\mathrm{P}_{\text {mean 3D-DSA }}=$ $189.3 \pm 99.13 \mathrm{~Pa} ; \mathrm{P}_{\text {mean } 4 \mathrm{D}-\mathrm{DSA}}=181.4 \pm 114.4 \mathrm{~Pa} ; r_{\mathrm{P} \text { systole }}=$ $\left.0.903, P<.001 ; r_{\mathrm{P} \text { diastole }}=0.842, P=.002\right)$. Regarding intraaneurysmal flow velocity, no significant difference between $3 \mathrm{D}$ and 4D-DSA was detected (systole: $\mathrm{V}_{3 \mathrm{D} \text {-DSA mean }}=80.8 \pm 50.94$ $\mathrm{mm} / \mathrm{s} ; \mathrm{V}_{4 \mathrm{D}-\mathrm{DSA}}{ }_{\text {mean }}=74.89 \pm 48.02 \mathrm{~mm} / \mathrm{s}$; diastole: $\mathrm{V}_{3 \mathrm{D}-\mathrm{DSA} \text { mean }}=31.61 \pm 22.2 \mathrm{~mm} / \mathrm{s} ; \mathrm{V}_{4 \mathrm{D}-\mathrm{DSA} \text { mean }}=29.9 \pm 21.45$ $\left.\mathrm{mm} / \mathrm{s} ; r_{\mathrm{V} \text { systole }}=0.930, P<.001 ; r_{\mathrm{V} \text { diastole }}=0.952, P<.001\right)$. See also Tables 1 and 2 for an overview.

Hemodynamics. Qualitative analysis of hemodynamics did not demonstrate differences between 3Dand 4D-DSA. Assessment of flow complexity ( simple 3D-DSA/4D-DSA $=2 / 2$, complex 3D-DSA/4D-DSA $=8 / 8$ ), flow stability (stable 3D-DSA/4D-DSA = $8 / 8$, unstable 3D-DSA/4D-DSA $=2 / 2$ ), and inflow concentration (diffuse $3 \mathrm{D}$ $\mathrm{DSA} / 4 \mathrm{D}-\mathrm{DSA}=4 / 4$, concentrated 3DDSA/4D-DSA $=6 / 6$ ) did not show any difference between readers. Analysis of WSS did not provide significant differences between 3D- and 4D-DSA in terms of AWSS (systole: AWSS $_{3 \mathrm{D}-\mathrm{DSA}}=$ $0.933 \pm 1.02 \mathrm{~Pa} ; \mathrm{AWSS}_{4 \mathrm{D}-\mathrm{DSA}}=$ $0.835 \pm 0.853 \mathrm{~Pa}$; diastole: $\mathrm{AWSS}_{3 \mathrm{D}-\mathrm{DSA}}=$ $0.26 \pm 0.28 \mathrm{~Pa} ; \mathrm{AWSS}_{4 \mathrm{D}-\mathrm{DSA}}=0.24 \pm$ $0.25 \mathrm{~Pa} ; r_{\text {AwsS }}$ systole $=0.936$; $P<.001 ; r_{\text {Awss }}$ diastole $=0.918$; $P<.001)$ and MWSS (systole: MWSS $_{3 \mathrm{D}-\mathrm{DSA}}=5.95 \pm 1.83 \mathrm{~Pa}$; $\mathrm{MWSS}_{4 \mathrm{D}-\mathrm{DSA}}=5.63 \pm 1.64 \mathrm{~Pa}$; diastole: MWSS $_{3 \mathrm{D}-\mathrm{DSA}}=1.84 \pm 0.73$ $\mathrm{Pa} ; \mathrm{MWSS}_{4 \mathrm{D}-\mathrm{DSA}}=1.78 \pm 0.69$ $\mathrm{Pa} ; r_{\text {MWss systole }}=0.927 ; P<.001$; $\left.r_{\text {Mwss diastole }}=0.879 ; P=.001\right)$. Calculation of impingement zones $\left(\mathrm{IZ}_{3 \mathrm{D}-\mathrm{DSA}}=\right.$ $12.4 \pm 14.2 \mathrm{~mm}^{2} ; \mathrm{IZ}_{4 \mathrm{D}-\mathrm{DSA}}=8.9 \pm 6.81$ $\left.\mathrm{mm}^{2} ; r_{\mathrm{IZ}}=0.957 ; P<.001\right)$ and lowstress zones $\left(\mathrm{LSZ}_{3 \mathrm{D}-\mathrm{DSA}}=254.6 \pm\right.$ $203.55 \mathrm{~mm}^{2} ; \mathrm{LSZ}_{4 \mathrm{D}-\mathrm{DSA}}=263.9 \pm$ $\left.199.08 \mathrm{~mm}^{2} ; r_{\text {LSZ }}=1.00 ; P=.01\right) \mathrm{did}$ not show significant differences. See Table 2 for an overview and Figs 3 and 4 for illustrative cases.

\section{DISCUSSION}

To date, 2D-DSA (in combination with 3D-DSA) is considered the criterion standard for visualization of the cerebral vasculature. As a novel imaging method, 4D-DSA offers the advantages of both modalities by providing not only timeresolved but also 3D images. Despite the acquisition time of 4D-DSA differing marginally from that of 3D-DSA ( 12 versus 5 seconds), total time and effort necessary for postprocessing 4D-DSA volumes are comparable with those of 3D-DSA and do not impair the clinical workflow. On the contrary, 4D-DSA might help reduce the amount of 2D-DSA series (eg, target projections).

Thus, 4D-DSA has the potential to reduce the dose and the amount of contrast medium necessary for cerebral conventional angiography, especially for the diagnostic work-up of complex neurovascular pathologies. Recent literature indicates a high level of reliability of 4D-DSA regarding qualitative parameters, ${ }^{5-8}$ yet knowledge of $4 \mathrm{D}$-DSA regarding quantitative parameters is still limited. Furthermore, injection protocols differ significantly between 3D- and 4D-DSA because time-resolved 4D-DSA datasets 


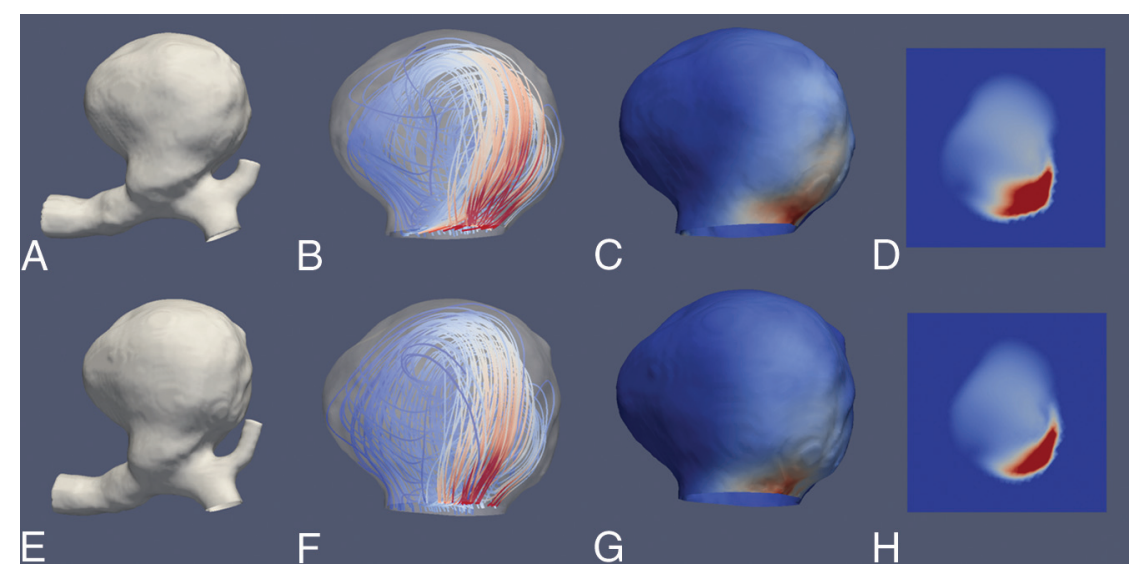

FIG 4. Illustrative case 2 . CFD simulations show a large saccular aneurysm of the carotid T. Upper row $(A-D)$ and lower row $(E-H)$ show reconstruction results derived from a 3D-DSA and a 4D-DSA dataset, respectively. $A$ and $E, 3 D$ view of the aneurysm using a volume-rendering technique and demonstrating comparable aneurysmal morphology. $B$ and $F$, Color-coded visualization of intraaneurysmal flow using streamlines (red and blue indicate high- and low-velocity magnitudes). The peak velocities show a predominant basal distribution. $C$ and $G$, In concordance, note maximum systolic wall shear stress (red and blue indicate high and low WSS) in the corresponding area. $D$ and $H$, Aneurysmal ostium, the associated flow conditions, and the match of areas with highvelocity magnitude (red and blue indicate high- and low-velocity magnitude). Overall, the congruence of hemodynamic characteristics between 3D- and 4D-DSA shows geometric accordance between the techniques.

are acquired using a dynamic injection protocol, whereas 3DDSA datasets are acquired by continuous injection of contrast medium. Therefore, we analyzed the ability of 4D-DSA to reproduce the vascular geometry in comparison with conventional 3D-DSA. On the one hand, we wanted to evaluate the applicability of 4D-DSA for performing CFD analysis. On the other hand, we wanted to use CFD itself for comparison of 3D- and 4D-DSA datasets with the help of the resulting (qualitative and quantitative) flow parameters.

In our series, all 3D- and 4D-DSA datasets were suitable for CFD analysis. Despite 4D-DSA yielding an insignificant underestimation of hemodynamic parameters compared with 3D-DSA in our series (that might refer to the different injection protocols), our analysis revealed that both qualitative and quantitative CFD parameters showed excellent agreement in the corresponding datasets. Therefore, we conclude that this consistency regarding CFD parameters derives from a consistent representation of aneurysmal geometry and that the dynamic injection protocol for acquisition of 4D-DSA does not influence or compromise the visualization of cerebral vasculature.

There is scant literature comparing the quantitative reliability of 4D-DSA with that of 2D and 3D. A single publication addresses detailed the quantitative accuracy of 4D-DSA in comparison with 2D-DSA: Lang et $\mathrm{al}^{8}$ evaluated 4D-DSA in 26 cases of cerebral aneurysms, AVMs, and dural arteriovenous fistulas regarding quantitative and qualitative parameters. Quantitative analysis of injection vessels, aneurysmal size, AVM nidus size, and the diameter of a main feeder of a fistula in 4D-DSA datasets demonstrated excellent agreement with 2D-DSA. Furthermore, qualitative evaluation of 4D-DSA was comparable with that of 2D-DSA. A comparison between 4D-DSA and 3D-DSA was not performed in their series though. Therefore, our data close the gap of knowledge regarding qualification of $4 \mathrm{D}-\mathrm{DSA}$ for $3 \mathrm{D}$ imaging of the cerebral vasculature. In line with the results of the excellent agreement between $4 \mathrm{D}$ - and 2D-DSA, our data demonstrate excellent agreement between $4 \mathrm{D}$ - and 3D-DSA.

So far, other authors studying $4 \mathrm{D}$ DSA have focused on qualitative comparisons of 4D-DSA with 3D- and 2DDSA. In this context, the publication of Sandoval-Garcia et $\mathrm{al}^{5}$ aroused our interest in the diagnostic utility of 4D-DSA compared with 2D- and 3D-DSA in the diagnosis of cerebrovascular abnormalities. Their analysis demonstrated in 26 cases of AVMs, dural arteriovenous fistulas, cerebral aneurysms, stenosis, and healthy individuals that the information content of 4D-DSA reconstructions is largely equivalent to that of the combination of 2D- and 3D-DSA. The specific comparison of 4D-DSA with 3D-DSA regarding quantitative parameters was not the object of this publication, yet the authors observed, concordant with our results, no significant disagreements between 4D-DSA and 2D-/ 3D-DSA concerning quantitative characteristics (eg, nidus size, aneurysm dimensions, and so forth).

Another publication of Sandoval-Garcia et $\mathrm{al}^{6}$ addressed the comparison of 4D-DSA with 2D- and 3D-DSA in the analysis of normal vascular structures in a canine model. The authors assessed, in 15 datasets, the qualification of 4D-DSA for analysis of the cerebral vasculature. The comparison among the 3 modalities was performed exclusively qualitatively though. 4D-DSA was rated superior to 2D- and 3D-DSA, respectively, and was regarded as the preferred method for vascular assessment. The authors did not observe any restriction or inferiority as a consequence of using 4D-DSA, corresponding to our results. Furthermore, the authors concluded that the use of 4D-DSA leads to a reduction in radiation and contrast doses.

\section{Limitations}

Although data analysis was successful in all 10 cases, our analysis has several limitations. First, it was limited by the small sample size and the rather mid-to-large size of the included aneurysms. Because the literature has shown the applicability of CFD analysis for small aneurysms as well, ${ }^{14}$ we conclude that our results are valid for not only mid-to-large size aneurysms but also small aneurysms. Second, our work covers only 1 type of vascular pathology (cerebral aneurysms) and only datasets acquired by selective injections either via the ICA or the vertebral artery. Comparable with other publications on CFD analyses, missing standardization for postprocessing is a relevant limitation. However, approximations and assumptions regarding blood viscosity and flow conditions are mandatory for CFD. ${ }^{10}$ In agreement with the experience of other authors, we could observe a high level of correlation between the hemodynamic patterns in our CFD models, the underlying raw data, and the corresponding 2D-DSA series. ${ }^{20}$ 


\section{CONCLUSIONS}

Despite a different injection protocol, 4D-DSA is a reliable basis for CFD analysis of the intracranial vasculature and provides equivalent visualization of intracranial aneurysm geometry compared with 3D-DSA.

Disclaimer: The concepts and results presented in this paper are based on research and are not commercially available.

Disclosures: Annette I. Birkhold-UNRELATED: Employment: Siemens. Jürgen Endres-UNRELATED: Consultancy: iSchemaview, Inc; Grant: Bayern Innovativ GmbH Projektträger Bayern-BayMED.* *Money paid to the institution.

\section{REFERENCES}

1. Wong SC, Nawawi O, Ramli N, et al. Benefits of 3D rotational DSA compared with 2D-DSA in the evaluation of intracranial aneurysm. Acad Radiol 2012;19:701-07 CrossRef Medline

2. Geers AJ, Larrabide I, Radaelli AG, et al. Patient-specific computational hemodynamics of intracranial aneurysms from 3D rotational angiography and CT angiography: an in vivo reproducibility study. AJNR Am J Neuroradiol 2011;32:581-86 CrossRef Medline

3. Hochmuth A, Spetzger U, Schumacher M. Comparison of threedimensional rotational angiography with digital subtraction angiography in the assessment of ruptured cerebral aneurysms. AJNR Am J Neuroradiol 2002;23:1199-205 Medline

4. Davis B, Royalty K, Kowarschik M, et al. 4D digital subtraction angiography: implementation and demonstration of feasibility. AJNR Am J Neuroradiol 2013;34:1914-21 CrossRef Medline

5. Sandoval-Garcia C, Yang P, Schubert T, et al. Comparison of the diagnostic utility of 4D-DSA with conventional 2D- and 3D-DSA in the diagnosis of cerebrovascular abnormalities. AJNR Am J Neuroradiol 2017;38:729-34 CrossRef Medline

6. Sandoval-Garcia C, Royalty K, Aagaard-Kienitz B, et al. A comparison of 4D-DSA with 2D and 3D-DSA in the analysis of normal vascular structures in a canine model. AJNR Am J Neuroradiol 2015;36: 1959-63 CrossRef Medline

7. Lescher S, Gehrisch S, Klein S, et al. Time-resolved 3D rotational angiography: display of detailed neurovascular anatomy in patients with intracranial vascular malformations. J Neurointerv Surg 2017; 9:887-94 CrossRef Medline

8. Lang S, Gölitz P, Struffert T, et al. 4D-DSA for dynamic visualization of cerebral vasculature: a single-center experience in 26 cases. AJNR Am J Neuroradiol 2017;38:1169-76 CrossRef Medline
9. Kallmes DF. Point: CFD-computational fluid dynamics or confounding factor dissemination. AJNR Am J Neuroradiol 2012;33: 395-96 CrossRef Medline

10. Cebral JR, Mut F, Weir J, et al. Association of hemodynamic characteristics and cerebral aneurysm rupture. AJNR Am J Neuroradiol 2011;32:264-70 CrossRef Medline

11. Zeng Z, Kallmes DF, Durka MJ, et al. Hemodynamics and anatomy of elastase-induced rabbit aneurysm models: similarity to human cerebral aneurysms? AJNR Am J Neuroradiol 2011;32: 595-601 CrossRef Medline

12. Steinman DA, Milner JS, Norley CJ, et al. Image-based computational simulation of flow dynamics in a giant intracranial aneurysm. AJNR Am J Neuroradiol 2003;24:559-66 Medline

13. Ren Y, Chen GZ, Liu Z, et al. Reproducibility of image-based computational models of intracranial aneurysm: a comparison between 3D rotational angiography, CT angiography and MR angiography. Biomed Eng Online 2016;15:50 CrossRef Medline

14. Cebral JR, Castro MA, Burgess JE, et al. Characterization of cerebral aneurysms for assessing risk of rupture by using patient-specific computational hemodynamics models. AJNR Am J Neuroradiol 2005;26:2550-59 Medline

15. Szikora I, Paal G, Ugron A, et al. Impact of aneurysmal geometry on intraaneurysmal flow: a computerized flow simulation study. $\mathrm{Neu}$ roradiology 2008;50:411-21 CrossRef Medline

16. Cebral JR, Castro MA, Appanaboyina S, et al. Efficient pipeline for image-based patient-specific analysis of cerebral aneurysm hemodynamics: technique and sensitivity. IEEE Trans Med Imaging 2005;24:457-67 CrossRef Medline

17. Kono K, Tomura N, Yoshimura R, et al. Changes in wall shear stress magnitude after aneurysm rupture. Acta Neurochir 2013; 155:1559-63 CrossRef Medline

18. Boegel M, Gehrisch S, Redel T, et al. Patient-individualized boundary conditions for CFD simulations using time-resolved 3D angiography. Int J Comput Assist Radiol Surg. 2016;11:1061-69 CrossRef Medline

19. Shojima M, Oshima M, Takagi K, et al. Magnitude and role of wall shear stress on cerebral aneurysm: computational fluid dynamic study of $\mathbf{2 0}$ middle cerebral artery aneurysms. Stroke 2004;35: 2500-05 CrossRef Medline

20. Cebral JR, Pergolizzi R Jr, Putman CM. Computational fluid dynamics modeling of intracranial aneurysms: qualitative comparison with cerebral angiography. Acad Radiol 2007;14:804-13 CrossRef Medline 